\title{
BMJ Open Infection-related complications after common infection in association with new antibiotic prescribing in primary care: retrospective cohort study using linked electronic health records
}

Birgitta van Bodegraven (D) , ${ }^{1}$ Victoria Palin, ${ }^{1}$ Chirag Mistry, ${ }^{1}$ Matthew Sperrin, ${ }^{1}$
Andrew White,${ }^{2}$ William Welfare ${ }^{3}$ Darren M Ashcroft, ${ }^{4}$ Tjeerd Pieter van Staa ${ }^{1,5}$

To cite: van Bodegraven B, Palin V, Mistry C, et al. Infection-related complications after common infection in association with new antibiotic prescribing in primary care: retrospective cohort study using linked electronic health records. BMJ Open 2021;11:e041218. doi:10.1136/ bmjopen-2020-041218

- Prepublication history and additional material for this paper is available online. To view these files, please visit the journal online (http://dx.doi.org/10. 1136/bmjopen-2020-041218)

Received 02 June 2020 Revised 17 November 2020 Accepted 29 December 2020

D) Check for updates

(c) Author(s) (or their employer(s)) 2021. Re-use permitted under CC BY-NC. No commercial re-use. See rights and permissions. Published by BMJ.

For numbered affiliations see end of article.

\section{Correspondence to} Birgitta van Bodegraven; birgitta.vanbodegraven@ manchester.ac.uk

\section{ABSTRACT}

Objective Determine the association of incident antibiotic prescribing levels for common infections with infection-related complications and hospitalisations by comparing high with low prescribing general practitioner practices.

Design retrospective cohort study Retrospective cohort study.

Data source UK primary care records from the Clinical Practice Research Datalink (CPRD GOLD) and SAIL Databank (SAIL) linked with Hospital Episode Statistics (HES) data, including 546 CPRD, 346 CPRD-HES and 338 SAIL-HES practices.

Exposures Initial general practice visit for one of six common infections and the proportion of antibiotic prescribing in each practice.

Main outcome measures Incidence of infection-related complications (as recorded in general practice) or infection-related hospital admission within 30 days after consultation for a common infection.

Results A practice with $10.4 \%$ higher antibiotic prescribing (the IQR) was associated with a $5.7 \%$ lower rate of infection-related hospital admissions (adjusted analysis, $95 \% \mathrm{Cl} 3.3 \%$ to $8.0 \%$ ). The association varied by infection with larger associations in hospital admissions with lower respiratory tract infection $(16.1 \% ; 95 \% \mathrm{Cl}$ $12.4 \%$ to $19.7 \%$ ) and urinary tract infection (14.7\%; $95 \%$ CI $7.6 \%$ to $21.1 \%$ ) and smaller association in hospital admissions for upper respiratory tract infection (6.5\%; $95 \% \mathrm{Cl} 3.5 \%$ to $9.5 \%$ ) The association of antibiotic prescribing levels and hospital admission was largest in patients aged $18-39$ years ( $8.6 \%$; $95 \% \mathrm{Cl} 4.0 \%$ to $13.0 \%)$ and smallest in the elderly aged $75+$ years $(0.3 \% ; 95 \% \mathrm{Cl}$ $-3.4 \%$ to $3.9 \%$ ).

Conclusions There is an association between lower levels of practice level antibiotic prescribing and higher infection-related hospital admissions. Indiscriminately reducing antibiotic prescribing may lead to harm. Greater focus is needed to optimise antibiotic use by reducing inappropriate antibiotic prescribing and better targeting antibiotics to patients at high risk of infection-related complications.

\section{Strengths and limitations of this study}

- Two large primary care databases with linked hospitalisation data were used to evaluate the difference in hospital admission after community-acquired common infections comparing high with low prescribing general practitioner practices.

- This analysis focuses on antibiotic prescribing at practice level with the emphasis on evaluating governmental guidance on reducing overall prescribing.

- Incidental antibiotic prescriptions without details on local antibiotic resistance levels were evaluated in this analysis, and the results can only be interpreted in this context.

- No data were extracted on infection severity or symptom scores; therefore, no conclusions can be drawn on the appropriateness of antibiotics prescribed.

\section{INTRODUCTION}

Common infections, such as sore throat or sinusitis, are often self-limiting and usually get better without antibiotics; nevertheless, they are frequently prescribed. ${ }^{12}$ Research regarding antimicrobial resistance (AMR) and antibiotic prescribing rates often focuses on reducing inappropriate prescribing to lower the threat of increasing AMR. ${ }^{3}$ Antibiotic prescribing for common self-limiting infections is often seen as a target for reduction. ${ }^{34}$ However, a proportion of common infections are caused by bacterial infections that may progress, and antibiotics may reduce infection-related adverse outcomes.

The UKAMR national action plan for 20192024 continues on from the last AMR strategy (2013-2018) with updated aims and targets to address the continued problem of resistance. One aim is to optimise antibiotic use through stewardship programmes, including a $25 \%$ 
reduction in antibiotic use in the community from the 2013 baseline. ${ }^{5}$ Antibiotic prescribing in primary care in England shows a declining trend $(-13.2 \%)$ between 2013 and 2017; however, to reach desired reduction targets, continued efforts are needed. ${ }^{3}$

A small number of studies have analysed the relationship between antibiotic prescribing rates and adverse events in primary care. Petersen $e t a l^{6}$ and Gulliford $e t a l^{7}$ studied the relationship between antibiotic prescribing rates in primary care and complication in patients with common respiratory tract infections (RTIs). Both studies reported reductions in incidence of pneumonia, as recorded by the general practitioner (GP), with higher levels of antibiotic prescribing. However, these studies did not evaluate the association of prescribing rates with the rate of hospital admission after common infections in primary care.

Gharbi $e t a l^{8}$ reported that prescribing immediate antibiotics in primary care to elderly patients for urinary tract infection (UTI) was associated with a lower risk of bloodstream infection, hospital admission and all-cause mortality compared with no antibiotics and deferred antibiotic prescribing. However, antibiotic prescribing in primary care is known to increase the risk of resistant infections. ${ }^{9}$ This highlights the challenge in balancing prescribing to reduce the risk of severe outcomes and limiting overall antibiotic consumption to slow the development of AMR.

The association between practice antibiotic prescribing rates and the rate of hospital admission after common infection when clearly separated from other infectionrelated complications managed in the community has not previously been studied. There is uncertainty with regards to the relationship between antibiotic prescribing levels and complications that can arise after various common infections. The objective of this study was to investigate the association between practice level antibiotic prescribing in primary care for multiple common infections and the rate of infection-related complications through comparison of high and low prescribing GP practices. These data provide insight into the role of antibiotic prescribing patterns in controlling the rate of adverse events.

\section{METHOD}

\section{Data sources}

The Clinical Practice Research Datalink (CPRD GOLD ${ }^{10}$ ) and the Secure Anonymised Information Linkage Databank (SAIL ${ }^{11}$ ) were used in this study. CPRD is a UK primary care database with routinely collected electronic health records. ${ }^{10}$ All patients registered with a participating general practice are anonymously included in the dataset. Data have been collected from 1987 and represents about $8 \%$ of the UK population. CPRD is broadly representative of the general UK population in terms of age, sex and ethnicity. ${ }^{10}$ The SAIL databank is a data repository of anonymised personal data collected for research from $75 \%$ of Welsh general practices. ${ }^{11}$ Within
SAIL, individual GP practices share anonymised patientlevel clinical information on symptoms, diagnoses and prescribed treatment. As Welsh GP practices are included in both CPRD and SAIL, they have been removed from CPRD to avoid replication.

For both data sources, all patient level data were aggregated up to practice level. The final CPRD dataset contained 546 GP practices of which 346 (located in England only) were linked with hospital admitted patient care data (Hospital Episode Statistics (HES)). The SAIL Databank included 338 GP practices, all linked to HES.

\section{Selection and eligibility criteria}

The CPRD study population included patients with a consultation between 1 January 2000 and 30 June 2015; for SAIL, the time period was between 1 January 2000 and 31 December 2017. The study population included patients with an initial GP consultation and clinical Read code for a common infection. This was defined as the first incident consultation for a common infection within 6 months and without an antibiotic prescription in the previous 1 month. Six common infections were included: upper respiratory tract infection (URTI; cough or cold, sore throat), lower respiratory tract infection (LRTI), otitis externa, otitis media, sinusitis and UTI.

Patients were eligible to be included if they were permanently registered at the GP practice, had a minimum of 1-year follow-up since data collection (except for children under one) and at least one record of an incident common infection. Male and females of any age were eligible. Patients were not required to have an antibiotic prescribed at the time of visit for common infection. Patients with an infection-related complication or an infection-related hospital admission in the 6 months prior or on the day of consultation were excluded. Individual patients were able to contribute multiple infection episodes, as long as the consultations were at least 6 months apart.

\section{Exposure and outcomes}

The number of patients who received an antibiotic at the consultation was determined. The practice antibiotic prescribing rate was the percentage of consultations that resulted in an antibiotic prescription in the complete study period.

Infection-related hospital admission was identified using the primary admission diagnosis using ICD-10 (International Classification of Diseases, Tenth Revision) codes from the linked HES data. This outcome was evaluated using the CPRD-HES and SAIL-HES datasets. The second outcome evaluated was infection-related complications as recorded in the primary care records identified from Read codes. Both outcomes were evaluated during the 30 days after the initial common infection consultation. In case of death or end of data collection within these 30 days, observations were censored. The outcomes were evaluated using the CPRD and SAIL datasets. 
Table 1 Demographic and characteristics of the GP practices included in CPRD, CPRD-HES and SAIL datasets

CPRD $n=546$

CPRD-HES linked $n=346$

SAIL $n=338$

\begin{tabular}{|c|c|c|c|}
\hline \multicolumn{4}{|l|}{ Consultations } \\
\hline Upper respiratory tract infection & 9646774 & 5698611 & 1956752 \\
\hline Lower respiratory tract infection & 2288616 & 1321593 & 435929 \\
\hline Otitis media & 864791 & 529946 & 215495 \\
\hline Sinusitis & 707736 & 422638 & 97636 \\
\hline Age (mean, SD) & $38.50(3.86)$ & $38.47(3.72)$ & $30.17(7.11)$ \\
\hline Sex female $(\%)$ & 58.98 & 59.06 & 56.25 \\
\hline \multicolumn{4}{|l|}{ Charlson comorbidity index (mean (\%)) } \\
\hline None (0) & 65.80 & 66.16 & 77.28 \\
\hline Very high $(>7)$ & 0.45 & 0.44 & 0.28 \\
\hline \multicolumn{4}{|l|}{ Region (count, \%) } \\
\hline North England & $109(20.0)$ & $83(24.0)$ & - \\
\hline Midlands & $120(22.0)$ & $87(25.1)$ & - \\
\hline South England & $158(28.9)$ & $124(35.8)$ & - \\
\hline London & $67(12.3)$ & $52(15.0)$ & \\
\hline $\begin{array}{l}\text { Devolved administrations (Northern Ireland } \\
\text { and Scotland) }\end{array}$ & $92(16.8)$ & - & - \\
\hline Wales & - & - & $338(100)$ \\
\hline Missing data & 37.32 & 1.09 & - \\
\hline Hospitalisation in previous year (mean (\%)) & 0.02 & 0.02 & 0.03 \\
\hline GPs per 1000 consults (mean, SD) & $3.54(2.30)$ & $3.52(2.25)$ & NA \\
\hline
\end{tabular}

GP count per 1000 consults was not available in SAIL databank.

CPRD, Clinical Practice Research Datalink; GP, general practitioner; HES, Hospital Episode Statistics; SAIL, Secure Anonymised Information Linkage.

Person time at risk was calculated for the registered CPRD and SAIL population by counting the days without diagnosis of infection-related complications during the 30-day follow-up after the date of common infection. The rates of infection-related outcomes were calculated by dividing the number of events by the person time at risk (per 1000 person-month). The outcomes were identified based on predefined code lists. Compiled code lists are available on clinicalcodes.org, ${ }^{12}$ and ICD-10 codes are available from van Staa et $a l .{ }^{13}$ The codes for outcomes and infections used were reviewed independently by two clinical epidemiologists. Infection-related hospital admission includes codes for admission such as for sepsis, endocarditis, acute RTI or bacterial meningitis. Infection-related complications as recorded in the primary care records includes any revisit to the GP for infection-related complications such as pneumonia, sepsis, quinsy, mastoiditis or meningitis in the 30-day follow-up period. The same set of conditions were included in both outcomes.

\section{Confounders}

The analysis was adjusted with the scaled mean at practice level of age, vaccination against influenza and hospital admission in the previous year. Additionally, the analysis 
Table 2 Rates of infection-related complications and or hospital admission in the 30 days after GP visit for common infection

\begin{tabular}{llll}
\hline Infection-related complications & $\begin{array}{l}\text { Number of cases (30-day } \\
\text { follow-up) }\end{array}$ & $\begin{array}{l}\text { Sum person-months } \\
\text { (30-day follow-up) }\end{array}$ & $\begin{array}{l}\text { Rate and 95\% CI } \\
\text { (per 1000 person- } \\
\text { month) }\end{array}$ \\
\hline $\begin{array}{l}\text { Infection-related complication GP recorded } \\
\quad \text { CPRD }\end{array}$ & 25721 & 19220606 & $1.34(1.32$ to 1.35) \\
\hline SAIL & 15192 & 3718739 & $4.09(4.02$ to 4.15$)$ \\
\hline Hospital admission & & & \\
\hline CPRD-HES linked & 17810 & 12335982 & $1.44(1.42$ to 1.47) \\
\hline SAIL-HES & 19796 & 3900897 & $5.08(5.00$ to 5.15) \\
\hline
\end{tabular}

GP-recorded infection-related complications were identified from the electronic health records, which included any revisit to the GP for complications after the initial consultation.

Hospital admission was identified from the linked HES data.

CPRD, Clinical Practice Research Datalink; HES, Hospital Episode Statistics; SAIL, Secure Anonymised Information Linkage.

was adjusted with the scaled proportion of each category at practice level of the following categorical characteristic: sex, Charlson Comorbidity Index, ${ }^{14}$ body mass index (BMI), smoking status (never, currently, past and unknown) and socioeconomic status (SES; least deprived to most deprived). The proportion of SES was derived from patients with linkage to Index of Multiple Deprivation (IMD) quintiles. Linked IMD data in quintiles based on patient's residential postcode were available for both datasets. Census based IMD data measures deprivation at area-level based on domains, such as income, employment, health, housing and general environment. ${ }^{15}$

Additionally, analyses using CPRD and CPRD-HES were adjusted with the mean at practice level of the number of GPs per 1000 consults, the patient transfer-out rate and region. No imputations or other adjustments were performed for missing characteristics in the covariates. Missing data were present for the following covariates: BMI (CPRD: 41.4\%), smoking status (CPRD: $30.4 \%$ ) and SES (CPRD: $37.3 \%$ ).

\section{Statistical analysis}

Infection-related complications were modelled with negative binomial regression using practice-level antibiotic prescribing as a predictor and the log of person time at risk as an offset. The unit of analysis is the practice. All variables were scaled with their associated IQR (IQR (interquartile range): 75 th-25th percentile) by dividing the original values by the IQR from the variable. ${ }^{16}$ This creates a natural comparison between high and low prescribing GP practices. The antibiotic prescribing rate was modelled continuously. Because of the scaling, the IQR becomes the unit that the effect size is expressed in. Both outcomes were compared against all common infections in the initial analysis. Models were adjusted for missing data using a covariate specific missing data indicator. The association of each of the six common infections was then studied against both outcomes separately. The analyses were further stratified by gender and age categories: $0-17,18-39,40-59,60-74$ and $75+$ years old to evaluate the varied prescribing among these risk groups.
The beta coefficient of the antibiotic prescribing rate was exponentiated and is presented as an incidence rate ratio (IRR). The effect estimates from the CPRD and SAIL cohorts were combined using a meta-analysis method with inverse variance weighting and DerSimonian and Laird random effect models.

Absolute difference in antibiotic prescribing between high and low prescribing practices was calculated from the prescribing rates (25th and 75th percentiles) and mean events per practice. The absolute difference in infection-related complications between high and low prescribing was calculated using the complication rate and the IRR. The number needed to treat (NNT) with antibiotics to prevent one event of hospital admission was calculated by dividing the absolute difference in antibiotic prescribing by the absolute difference in complications. Forestplot, ${ }^{17}$ dplyr $^{18}$ and MASS ${ }^{19}$ packages in R were used for the analysis. All analyses were performed using $\mathrm{R}$ software V.3.4.1 (R Foundation for Statistical Computing; Vienna, Austria).

\section{Patient and public involvement}

No patients were involved in the study design, and no patients were asked to consult on the outcomes or interpretation of the results. Results will be disseminated to relevant patient communities through news media and social media.

\section{RESULTS}

The study was based on a total of 19.6 million GP consultations for common infections. URTI was the most frequent common infection (CPRD: 9646 774) followed by LRTI (CPRD: 2288 616) and UTI (CPRD: 1511 176). A total of 884 GP practices were included in the analysis (CPRD: 546; SAIL: 338) (table 1). The mean age of the practice population was 38 years in CPRD and 30 years in SAIL. The majority of patients had no comorbidities recorded (Charlson score: 0 ). There were 25721 cases of infectionrelated complications as recorded in primary care in CPRD and 15192 cases in SAIL. The rate of these complications 
Table 3 Antibiotic prescribing rates for each common infection across practices included in CPRD ( $n=546)$, CPRD-HES $(n=346)$ and SAIL $(n=338)$.

\begin{tabular}{|c|c|c|c|c|c|c|}
\hline & Mean \% (SD) & $5 \%$ & $25 \%$ & $50 \%$ & $75 \%$ & $95 \%$ \\
\hline \multicolumn{7}{|c|}{$\begin{array}{l}\text { Upper respiratory tract infection (URTI); } \\
\text { URTI, cough or cold, sore throat }\end{array}$} \\
\hline CPRD & $46.14(11.71)$ & 28.59 & 38.25 & 45.14 & 53.73 & 66.36 \\
\hline CPRD-HES linked & $43.74(10.97)$ & 28.88 & 38.17 & 45.15 & 53.09 & 63.97 \\
\hline SAIL & 43.37 (12.07) & 24.83 & 34.57 & 42.88 & 51.76 & 63.43 \\
\hline \multicolumn{7}{|c|}{$\begin{array}{l}\text { Lower respiratory tract infection; } \\
\text { Excluding community acquired pneumonia }\end{array}$} \\
\hline CPRD & $84.79(8.89)$ & 69.79 & 81.45 & 86.68 & 90.52 & 94.40 \\
\hline CPRD-HES linked & $85.24(8.03)$ & 70.89 & 81.90 & 86.80 & 90.57 & 94.68 \\
\hline SAIL & $78.11(11.66)$ & 55.47 & 71.56 & 80.45 & 86.69 & 93.17 \\
\hline \multicolumn{7}{|l|}{ Otitis externa } \\
\hline CPRD & $26.33(8.98)$ & 15.34 & 20.00 & 24.55 & 31.00 & 42.70 \\
\hline CPRD-HES linked & $26.52(8.44)$ & 15.34 & 20.13 & 25.16 & 31.37 & 41.57 \\
\hline SAIL & 29.57 (10.65) & 14.92 & 22.03 & 28.71 & 34.89 & 48.5 \\
\hline \multicolumn{7}{|l|}{ Otitis media } \\
\hline CPRD & $78.10(10.86)$ & 58.35 & 73.05 & 80.27 & 86.09 & 91.57 \\
\hline CPRD-HES linked & $78.27(9.83)$ & 59.20 & 73.35 & 79.51 & 85.81 & 91.30 \\
\hline SAIL & $78.49(11.81)$ & 54.91 & 72.64 & 80.57 & 87.49 & 92.65 \\
\hline \multicolumn{7}{|l|}{ Sinusitis } \\
\hline CPRD & $84.97(8.93)$ & 67.89 & 82.48 & 87.13 & 90.29 & 94.43 \\
\hline CPRD-HES linked & $85.75(7.88)$ & 70.07 & 83.20 & 87.60 & 90.63 & 94.57 \\
\hline SAIL & $82.12(9.91)$ & 63.36 & 77.44 & 84.22 & 88.89 & 94.73 \\
\hline \multicolumn{7}{|l|}{ Urinary tract infection } \\
\hline CPRD & $85.90(7.39)$ & 74.01 & 82.96 & 87.28 & 90.98 & 93.72 \\
\hline CPRD-HES linked & $86.06(6.40)$ & 74.08 & 83.19 & 87.01 & 90.79 & 93.30 \\
\hline SAIL & 81.50 (10.30) & 61.46 & 76.70 & 84.66 & 88.65 & 93.18 \\
\hline
\end{tabular}

Rates are presented for six common infections. Proportion of consultations with antibiotics prescribed is presented with the mean percentage and the fifth to 95th percentile at practice level. The mean percentage of antibiotic prescribed in CPRD after a consultation for URTI was $46.1 \%$.

CPRD, Clinical Practice Research Datalink; HES, Hospital Episode Statistics; SAIL, Secure Anonymised Information Linkage.

was 1.3 and 4.1 per 1000 person-months, respectively. For infection-related hospital admission, the number of cases was 17810 in CPRD-HES and 19796 in SAIL-HES, with rates of 1.4 and 5.1 per 1000 person-months, respectively (table 2). The majority of antibiotics were prescribed for LRTI, sinusitis and UTI (table 3). Antibiotics were less likely to be prescribed for otitis externa. There was considerable variability between general practices in the percentages of patients prescribed an antibiotic. For URTI, 28.6\% of the patients received an antibiotic at the fifth percentile practice and $66.4 \%$ at the 95 th percentile practice. Summary counts of infection-related hospital admission types from CPRD-HES are available in online supplemental appendix 1.

\section{Infection-related hospital admission}

The incidence of infection-related hospital admission was found to be associated with the practice-level antibiotic prescribing rate (figure 1). A $10.4 \%$ higher antibiotic prescribing rate (IQR) was associated with an IRR of 0.943 (95\% CI $0.920-0.967$ ), denoting a $5.7 \%$ lower infection-related hospital admission rate in the combined analysis. Results between CPRD-HES and SAIL-HES were comparable. In CPRD-HES, a $10.1 \%$ higher antibiotic prescribing rate was associated with an IRR of $0.959(95 \%$ CI 0.926-0.992), meaning a $4.1 \%$ lower hospital admission rate. For SAIL-HES, this was $7.2 \%$ (IRR: $0.928 ; 95 \%$ CI 0.895 to 0.961 ) lower with the IQR of $10.7 \%$ higher antibiotic prescribing by GP practices.

The observed association varied by infection. In the combined analysis, the largest association was observed in LRTI (IRR: 0.839 (16.1\%); 95\% CI 0.803 to 0.876 ), UTI (IRR: 0.853 (95\% CI 0.788 to 0.924$) ; 14.7 \%)$ and URTI (IRR: 0.935 (95\% CI 0.905 to 0.965$) ; 6.5 \%$ ) (figure 2 ). In patients with URTI, $14.9 \%$ (CPRD-HES) and $17.2 \%$ 


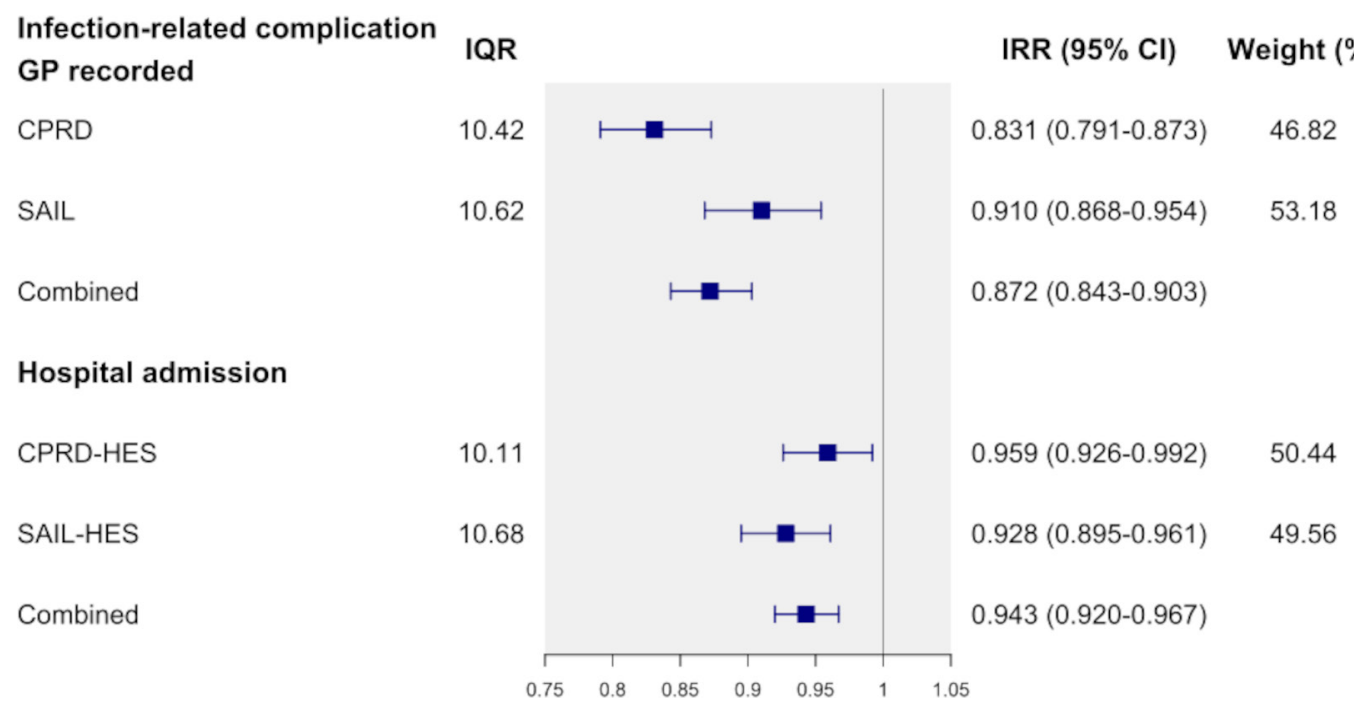

Figure 1 Incidence rate ratios and $95 \% \mathrm{Cl}$ of GP-recorded infection-related complications and hospital admissions comparing antibiotic prescribing at 75th to 25th percentile (IQR). Results are presented by data source. CPRD and SAIL effect estimates were combined using a frandom effect meta-analysis method. CPRD, Clinical Practice Research Datalink; HES, Hospital Episode Statistics; SAIL, Secure Anonymised Information Linkage.

(SAIL-HES), higher antibiotic prescribing was associated with infection-related hospital admissions being lower by $7.7 \%(0.923 ; 95 \%$ CI 0.879 to 0.969$)$ and $5.6 \%(0.944$; $95 \%$ CI 0.905 to 0.984$)$. LRTI was associated with a $14.2 \%$ (CPRD-HES, IRR: $0.858 ; 95 \%$ CI 0.808 to 0.911 ) and $18.2 \%$ (SAIL-HES, IRR: 0.818 ; $95 \%$ CI 0.767 to 0.872 ) lower incidence for hospital admission when antibiotic prescribing was higher by $8.7 \%$ and $15.1 \%$. In patients who consulted their GP for UTI, the incidence of hospital admission was $10.5 \%$ (IRR: 0.895 (95\% CI 0.783 to 1.027 ) lower with $7.6 \%$ higher antibiotic prescribing (CPRD-HES). In SAIL-HES, $12.0 \%$ higher antibiotic prescribing for UTI was associated with lower incidence by $16.8 \%$ (IRR: 0.832 (95\% CI 0.755 to 0.919$)$ ). Patients aged $18-39$ years old had the largest association for hospital admission (CPRD-HES: 0.884 (95\% CI 0.823 to 0.949 ; IQR unit: 10.88)) among the age categories (figure 3 ).

The number needed to treat with antibiotics to prevent one patient from developing infection-related complications was calculated over the 30 -day follow-up period. The number needed to treat for patients with URTI at risk of hospital admission was 1164. For patients with LRTI and UTI, the number needed to treat was 417 and 484 , respectively.

\section{GP-recorded infection-related complications}

Higher levels of antibiotic prescribing by GP practices were associated with lower incidence of infection-related complication as recorded by the GP. The incidence of GP-recorded infection-related complications reduced by $16.9 \%$ (0.831; $95 \%$ CI 0.791 to 0.873$)$ and $9.0 \%$ (0.910; $95 \%$ CI 0.866 to 0.954 ) with an increase in antibiotic prescribing of $10.4 \%$ and $10.6 \%$ for CPRD and SAIL, respectively.

Evaluating the observed association by common infection separately found that URTI was associated with lower GP-recorded infection-related complications by $20.4 \%(0.803 ; 95 \%$ CI 0.758 to 0.852$)$ when antibiotic prescribing increased by $15.5 \%$ in CPRD. In SAIL, the observed reduction was $12.7 \%(0.873 ; 95 \%$ CI 0.832 to 0.916 ) when antibiotic prescribing increased by $17.2 \%$.

Antibiotic prescribing for LRTI being higher by $9.1 \%$ and $15.1 \%$ was associated with the incidence of GP-recorded infection-related complications being lower by 16.2\% (IRR: $0.838 ; 95 \%$ CI 0.786 to 0.895 ) and $5.5 \%$ (IRR: $0.945 ; 95 \%$ CI 0.871 to 1.027 ) for CPRD and SAIL, respectively. For UTI, the incidence of GP-recorded infectionrelated complications was similarly lowered across CPRD (15.6\%; 0.844 (95\% CI 0.770 to 0.926 ) (IQR unit: 8.01)) and SAIL (8.7\%; 0.913 (95\% CI 0.838 to 0.997 ) (IQR unit: 11.95)).

No effect modification by gender was observed in any of the datasets evaluated (figure 3). The effect was more obvious in younger patients. Patients aged $0-17$ years had the largest association in GP-recorded infection-related complications in CPRD (22\%; IRR: 0.780 (95\% CI 0.725 to 0.839$)$; IQR: 12.05). Patients aged $0-17$ years and 40-59 years showed similar associations for both datasets (figure 3). Polynomials were fitted on a deciled antibiotic prescribing rate as a sensitivity analysis. First order polynomials best fitted the data and showed a downward linear trend from low to high prescribing (online supplemental appendix 2). An inverse association was found in an additional sensitivity analysis which paired URTI and LRTI with plausible subsequent infection-related complications, such as pneumonia and hospital admission for LRTI (online supplemental appendix 3. In patients who consulted their GP for LRTI, the incidence of a hospital admission with LRTI was $18 \%(0.820$ (95\% CI 0.765 to $0.879)$ ) lower with $8.7 \%$ higher antibiotic prescribing (CPRD-HES). 


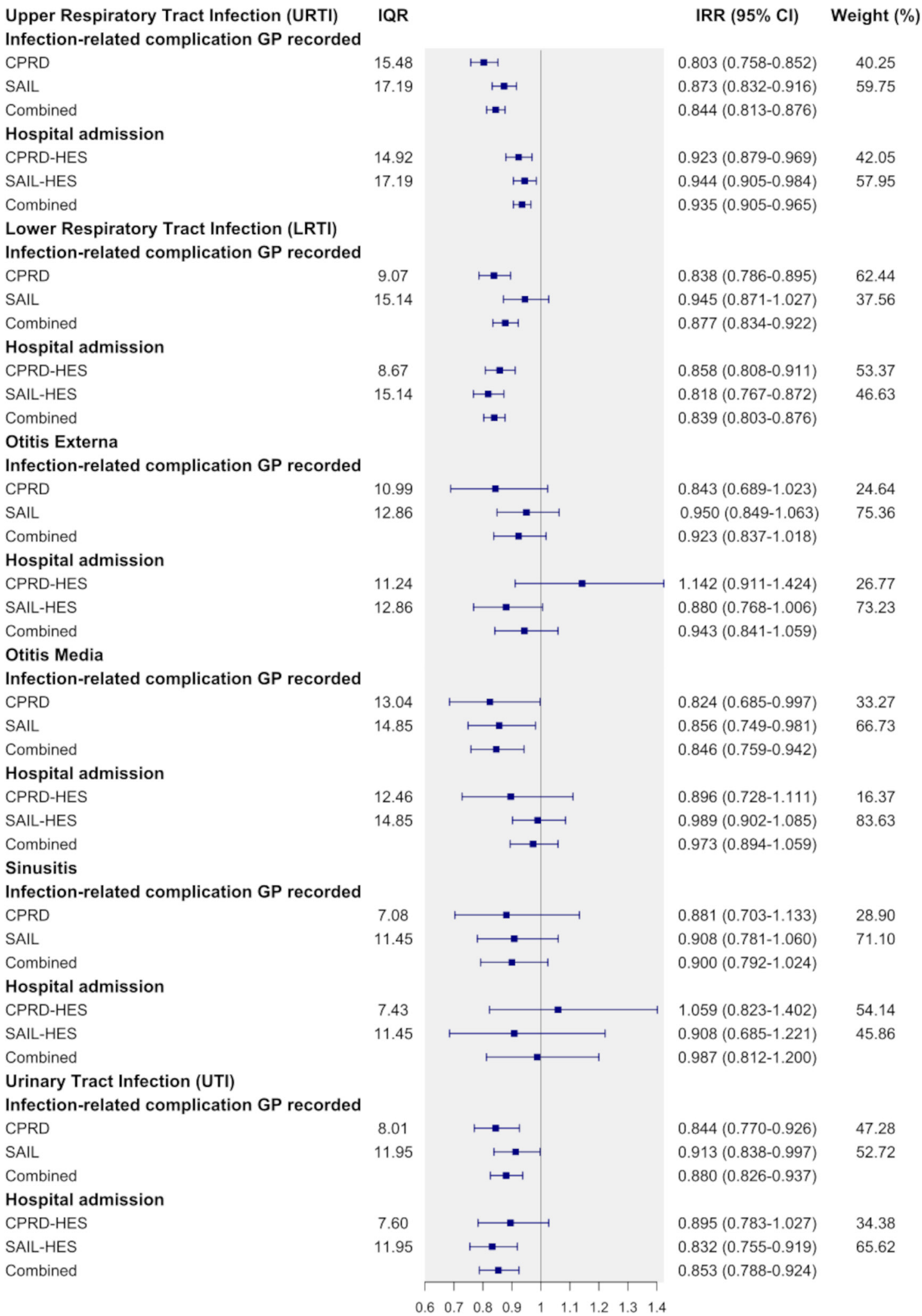

Figure 2 Effect estimates (IRRs and 95\% Cl) of GP-recorded infection-related complications and hospital admissions. Analyses compared antibiotic prescribing at 75th and 25th percentile (IQR) by six common infections. The IRR for hospital admission after a consultation for URTI in CPRD-HES was 0.923 . This means for a $14.9 \%$ increase in antibiotic prescribing the rate of hospital admission is reduced by 7.7\%. CPRD, Clinical Practice Research Datalink; GP, general practitioner; HES, Hospital Episode Statistics; SAIL, Secure Anonymised Information Linkage.

\section{DISCUSSION}

This study found that higher levels of incident antibiotic prescribing by practices were associated with lower rates of hospital admission and GP diagnosed infection-related complications. Lower rate of poor clinical outcomes with higher levels of antibiotic prescribing was more 


\section{Infection-related complication GP recorded Gender Male}

CPRD

SAIL

Combined

Gender Female

CPRD

SAIL

Combined

Hospital admission

Gender Male

CPRD-HES

SAIL-HES

Combined

Gender Female

CPRD-HES

SAIL-HES

Combined

\section{Infection-related complication GP recorded \\ Age 0-17}

CPRD

SAIL

Combined

Age 18-39

CPRD

SAIL

Combined

Age 40-59

CPRD

SAIL

Combined

Age 60-74

CPRD

SAIL

Combined

Age 75+

CPRD

SAIL

Combined

Hospital admission

Age 0-17

CPRD-HES

SAIL-HES

Combined

Age 18-39

CPRD-HES

SAIL-HES

Combined

Age 40-59

CPRD-HES

SAIL-HES

Combined

Age 60-74

CPRD-HES

SAIL-HES

Combined

Age 75+

CPRD-HES

SAIL-HES

Combined
IQR

10.84

11.12

10.26

9.80

10.65
11.12


9.74
9.80

12.05

11.26

10.91

11.20

10.2

10.95

9.31
9.55

10.01

11.20

(1)

11.68

11.26

10.88

10.88
11.20

9.65

9.65
10.95

(9.10

9.10
9.55

9.57

11.20
IRR (95\% Cl)

$0.809(0.767-0.854)$ $0.935(0.887-0.986)$

$0.872(0.839-0.905)$

$0.848(0.805-0.894) \quad 45.59$

$0.888(0.845-0.932) \quad 54.41$

$0.869(0.839-0.901)$

$0.972(0.932-1.014) \quad 46.36$

$0.943(0.906-0.980) \quad 53.64$

$0.956(0.929-0.984)$

$0.954(0.915-0.995) \quad 47.01$

$0.913(0.877-0.950) \quad 52.99$

$0.932(0.906-0.959)$

$0.780(0.725-0.839)$

$0.916(0.874-0.960)$

$0.874(0.840-0.910)$

$0.814(0.764-0.868) \quad 56.45$

$0.867(0.805-0.933) \quad 43.55$

$0.837(0.798-0.878)$

$0.824(0.777-0.874) \quad 65.31$

$0.937(0.864-1.016) \quad 34.69$

$0.862(0.822-0.904)$

$0.806(0.757-0.858) \quad 58.52$

$0.957(0.888-1.032) \quad 41.48$

$0.865(0.825-0.908)$

$0.871(0.809-0.937) \quad 58.00$

$0.949(0.871-1.034) \quad 42.00$

$0.903(0.854-0.955)$

$0.956(0.896-1.020) \quad 23.49$

$0.962(0.928-0.997) \quad 76.51$

$0.961(0.931-0.991)$

$0.884(0.823-0.949) \quad 48.39$

$0.942(0.880-1.010) \quad 51.61$

$0.914(0.870-0.960)$

$0.968(0.907-1.033) \quad 57.79$

$0.894(0.829-0.964) \quad 42.21$

$0.936(0.891-0.983)$

$0.988(0.934-1.046) \quad 59.96$

$0.925(0.863-0.991) \quad 40.04$

$0.962(0.921-1.005)$

$1.014(0.964-1.066) \quad 53.45$

$0.980(0.929-1.034) \quad 46.55$

$0.997(0.961-1.034)$

Figure 3 Association of GP-recorded infection-related complications and hospital admissions comparing practice antibiotic prescribing at 75th and 25th percentile (IQR) by gender and age groups. Weights are from random effects analysis. CPRD, Clinical Practice Research Datalink; GP, general practitioner; HES, Hospital Episode Statistics; SAIL, Secure Anonymised Information Linkage.

pronounced for URTI, LRTI and UTI but had no association with poor outcomes for otitis media and otitis externa. A higher level of incident antibiotic prescribing in younger patients was associated with better clinical outcomes, while no association was observed in patients over 40 years old. 
This is the first study to use two large primary care databases with linked hospitalisation data to evaluate the difference in hospital admission after common infections comparing high with low prescribing GP practices. The focus of this analysis was at practice level with the emphasis on evaluating governmental guidance on reducing overall prescribing. Practice-level prescribing proportion as a standardised antibiotic measure allows for comparing the range of GP prescribing within and between datasets with similar inclusion criteria. Other standard measures, such as age-adjusted and sex-adjusted STAR-PU (Specific Therapeutic group Age-sex Related Prescribing Unit) prescribing units, are available, although the research question here specifically focused on the reduction of overall antibiotic prescribing levels regardless of patient-mix within a practice. The study population was restricted to new antibiotic prescribing in patients with newly developed common infections. Including patients with more complex clinical scenarios, like repeated antibiotic users, complicates the estimation of the effect of interest. Past consultations and potential treatment for a common infection may be associated with future consultations, treatment and future outcomes of interest. This will lead to a problem when the outcome of interest cannot be related back to a single index visit and instead potentially to more than one visit. The results of this analysis can only be interpreted in the context of the incidental antibiotic user.

This practice-level analysis possibly simplifies the relationship between antibiotic prescribing rate and infection-related complications by aggregating data up to practice level and ignoring diversity in patient characteristics within a practice. Some potential confounding at practice level may occur due to variation in patient population frailty even when characteristics have been accounted for at practice level. ${ }^{20}$ In addition, although this analysis attempted to adjust for several available factors that might influence the association investigated, missing data were present in some of the covariates. The analyses accounted for this by using a missing indicator and the presence of missing data in the covariates could have influenced the estimates, although the large sample size and replication of the analysis in a second database (SAIL) gives weight to the interpretation of the results. There remains a potential for additional residual confounding by non-available covariates or other factors, such as quality of care, access to GPs and practices and availability of consultations, all of which have been linked to deprivation. ${ }^{21} 22$ However, without specific knowledge of a physician's prescribing preference relative to guidance, or qualitative data regarding patient care, it is not possible to evaluate the effects of these factors on the observed prescribing levels. Diagnoses are based on clinical coding both in primary and secondary care and potential misclassifications or misdiagnoses in the underlying data could have occurred. Differences in coding practices for common infections among English GP practices has been evaluated previously and found to be problematic at times. ${ }^{4}$ As no data were available on infection severity or symptom scores, no conclusions can be drawn on the appropriateness of antibiotics prescribed. This analysis was based on digital patient charts without access to free text due to GDPR rules as this poses a possible patient identification risk. Digital patient charts are automatically generated and transferred to the database. In addition, a small proportion of prescribing may be attributable to out of hours prescribing where coding of these consultation or prescriptions into the patient's record is performed afterwards and therefore subject to error and misclassification, potentially leading to an overestimation of the observed association.

The incidence rates of the clinical outcomes were different between SAIL and CPRD, with higher rates in Wales. There has been a measles epidemic in Wales recently that may partly explain these differences. However, this remains speculative. Infections are often localised and infection rates differ between locations. In addition, another possible explanation could be that this difference is due to coding behaviour. However, the level of data available does not allow in-depth investigation into this difference. The NNTs presented are related to the 30-day follow-up window. They may appear large and initial clinical relevance uncertain. UK guidance for initiating statin use states those with a 10-year risk of $10 \%-19 \%$ are eligible. Converting this 10 -year risk to a 30-day estimated NNT gives an NNT of $1139(10 \%)$ and $569(19 \%)$. These NNTs are similar to those presented in this analysis and have led to a change in clinical practice and prescribing behaviour.

Those with weaker immune systems, the very young and very old, have an elevated susceptibility to infections that may increase their antibiotic use and risk of related complications. ${ }^{23}$ Analysis performed by age group showed that higher levels of antibiotic prescribing were associated with reduced infection-related complications in younger patients. Higher levels of antibiotic prescribing were not associated with lower rates of infection-related complications in patients aged $60+$ years. A possible explanation for this is that increased lifetime exposure and repeated use of antibiotics could reduce antibiotic effectiveness, for example, due to altered pharmacokinetics. ${ }^{24}$ Recent research reported reduced effectiveness of antibiotics with repeated use over several years. ${ }^{13} \mathrm{~A}$ literature review by Costelloe $e t$ a ${ }^{\ominus}$ found that individuals who were prescribed an antibiotic for respiratory or UTIs develop bacterial resistance that was detectable for up to 12 months. Similar association has been reported recently for resistant blood stream infection after UTI prescribing. ${ }^{25}$ However, further research is needed to assess any age effect in the effectiveness of antibiotics. Another reason may be that GPs may be more hesitant to withhold antibiotics from older patients to avoid under-treatment, leading to seeing a greater response in younger patients at higher prescribing rates.

The more antibiotics prescribed, the higher the GP reattendance rates for common infections and subsequently 
the larger the represcribing antibiotic rate becomes. ${ }^{26} \mathrm{~A}$ randomised trial involving 34 general practices following the STAR educational programme saw reductions in overall levels of antibiotic prescribing in the intervention group. ${ }^{27}$ Hospital admission for RTIs and complications increased by $1.9 \%$ in the intervention group, suggesting that reduced antibiotic prescribing may increase hospital admission. However, this result was not found to be statistically different and had limited statistical power.

UK initiatives have included the TARGET toolkit and the Quality Premium (QP) to reduce overall levels of antibiotic use. ${ }^{27-29}$ The QP was introduced in April 2015 and provided a financial incentive to Clinical Commissioning Groups to reduce antibiotic prescribing rates. A significant 3\% reduction in antibiotic prescribing rate was observed after this initiative was introduced, with greatest reduction in children. ${ }^{30}$ Reducing antibiotic prescribing rates may be good for antibiotic resistance but as shown here could potentially cause more infection-related complications. Antibiotic prescribing requires a careful balance; with each prescription to treat and reduce the risk of infection-related complications, the chance of developing resistant infections increases for individual patients and drives AMR risk for the wider community. With the current aim to reduce antibiotic prescribing in the community in the UK by $25 \%$ from the 2013 baseline, particular focus is required to understand individual patient risk, reducing inappropriate prescribing and monitor infection-related complications. For patients with LRTI in primary care, Moore et $a l^{31}$ modelled a predictive value of the risk of patients developing serious outcomes including hospital admission. Such a direct approach, together with delayed prescribing strategies ${ }^{32}$ are suggested to target prescribing to those most likely to develop complications and reduce overall prescribing.

A Cochrane review of 27 trials on antibiotics for sore throat found that antibiotics prevented complications (acute rheumatic fever, glomerulonephritis, otitis media and sinusitis) in patients (NNT to benefit=200), but the rate of complications was low (approximately $0.7 \%$ ) and the benefit of antibiotic prescribing may not always be clear. ${ }^{33}$ Similarly, another Cochrane review focused on antibiotics for acute otitis media in children found that serious complications, such as mastoiditis and meningitis, were rare $(3 / 3000$ children $) .{ }^{34}$ Both reviews highlighted the inability to predict which patients are at risk of developing complications. Clinical tools such as the FeverPAIN score and Centor criteria are used to guide antibiotic treatment for acute sore throat. However, Little et $a l^{35}$ concluded that clinical scores such as FeverPAIN were of limited value in predicting clinical complications.

In conclusion, lower levels of practice level antibiotic prescribing were associated with higher levels of infectionrelated complications and hospital admissions. Identifying and developing accurate clinical tools for predicting which patients are at risk of complications requires much needed further research. To improve patient outcomes and reduce the risk of avoidable complications, there is a need to target patients most likely to benefit from effective, safe prescribing, based on shared decision making.

\section{Author affiliations}

${ }^{1}$ Health e-Research Centre, School of Health Sciences, Faculty of Biology, Medicine and Health, The University of Manchester, Manchester, UK

${ }^{2}$ NHS Greater Manchester Shared Service, Oldham, UK

${ }^{3}$ Public Health England North West, Manchester, UK

${ }^{4}$ Centre for Pharmacoepidemiology and Drug Safety, NIHR Greater Manchester Patient Safety Translational Research Centre, School of Health Sciences, Faculty of Biology, Medicine and Health, Manchester Academic Health Science Centre (MAHSC), The University of Manchester, Manchester, UK

${ }^{5}$ Utrecht Institute for Pharmaceutical Sciences, Utrecht University, Utrecht, the Netherlands

Correction notice This article has been corrected since it first published. The provenance and peer review statement has been included.

Contributors BvB and TvS contributed to the idea and design of the study. TvS extracted the relevant data from the databases. BvB analysed and interpreted the data with feedback from TvS and MS. BvB drafted the initial paper. VP, CM, MS, AW, WW and DA contributed to drafts and critical revision for intellectual content. All authors approved the final manuscript.

Funding This work was supported by Connected Health Cities, which is a Northern Health Science Alliance led programme funded by the Department of Health and Social Care and delivered by a consortium of academic and National Health Service (NHS) organisations across the north of England. This study is partly based on data from the Clinical Practice Research Datalink obtained under licence from the UK Medicines and Healthcare products Regulatory Agency (MHRA). Hospital Episode Statistics (HES) data are subject to Crown copyright (2018) protection, reused with the permission of The Health, \& Social Care Information Centre, all rights reserved. The data are provided by patients and collected by the NHS as part of their care and support. This study also used anonymised data held in the Secure Anonymised Information Linkage (SAIL) System, which is part of the national e-health records infrastructure for Wales.

Disclaimer The interpretation and conclusions contained in this study are those of the authors alone, and not necessarily those of the SAIL, MHRA, NHSA (Northern Health Science Alliance), NHS or the Department of Health.

Competing interests All authors have completed the ICMJE uniform disclosure form and declare: BvB, VP, CM, DA and TvS report grants from the Department of Health and Social Care for the submitted work; no financial relationships with any organisations that might have an interest in the submitted work in the previous three years; no other relationships or activities that could appear to have influenced the submitted work.

Patient consent for publication Not required.

Ethics approval The study protocol was approved by the Independent Scientific Advisory Committee for Clinical Practice Research Datalink (CPRD) research (protocol number 16_153) and SAlL's Information Governance Protocol Review Panel (protocol number 0693). We would like to acknowledge all the data providers who make anonymised data available for research.

Provenance and peer review Not commissioned; externally peer reviewed.

Data availability statement Data may be obtained from a third party and are not publicly available. Read codes used are published on Clinicalcodes.org. Electronic health records are, by definition, considered sensitive data in the UK by the Data Protection Act and cannot be shared via public deposition because of information governance restriction in place to protect patient confidentiality. Access to data is available only once approval has been obtained through the individual constituent entities controlling access to the data. CPRD data can be requested via application to the Clinical Practice Research Datalink (www.cprd.com), and SAIL data are available by application to the Secure Anonymised Information Linkage Databank (https://saildatabank.com/).

Supplemental material This content has been supplied by the author(s). It has not been vetted by BMJ Publishing Group Limited (BMJ) and may not have been peer-reviewed. Any opinions or recommendations discussed are solely those of the author(s) and are not endorsed by BMJ. BMJ disclaims all liability and responsibility arising from any reliance placed on the content. Where the content includes any translated material, BMJ does not warrant the accuracy and reliability of the translations (including but not limited to local regulations, clinical guidelines, 
terminology, drug names and drug dosages), and is not responsible for any error and/or omissions arising from translation and adaptation or otherwise.

Open access This is an open access article distributed in accordance with the Creative Commons Attribution Non Commercial (CC BY-NC 4.0) license, which permits others to distribute, remix, adapt, build upon this work non-commercially, and license their derivative works on different terms, provided the original work is properly cited, appropriate credit is given, any changes made indicated, and the use is non-commercial. See: http://creativecommons.org/licenses/by-nc/4.0/.

\section{ORCID iD}

Birgitta van Bodegraven http://orcid.org/0000-0002-8359-9017

\section{REFERENCES}

1 National Insitute for Health and Care Excellence (NICE), NHS, Centre for Clinical Practice at NICE, 2008. Respiratory tract infections (selflimiting): prescribing antibiotics. Available: https://www.nice.org.uk/ guidance/cg69/chapter/1-guidance

2 Pouwels KB, Dolk FCK, Smith DRM, et al. Actual versus 'ideal' antibiotic prescribing for common conditions in English primary care. $J$ Antimicrob Chemother 2018;73:19-26.

3 Public Health England. English surveillance programme for antimicrobial utilisation and resistance (ESPAUR) report 2018, 2018. Available: https://www.gov.uk/government/publications/englishsurveillance-programme-antimicrobial-utilisation-and-resistanceespaur-report

4 Smieszek T, Pouwels KB, Dolk FCK, et al. Potential for reducing inappropriate antibiotic prescribing in English primary care. $J$ Antimicrob Chemother 2018;73:ii36-43.

5 Department of Health and Social Care. UK 5-year action plan for antimicrobial resistance 2019 to 2024, 2019. Available: https:// assets.publishing.service.gov.uk/government/uploads/system/ uploads/attachment_data/file/784894/UK_AMR_5_year_national_ action_plan.pdf

6 Petersen I, Johnson AM, Islam A, et al. Protective effect of antibiotics against serious complications of common respiratory tract infections: retrospective cohort study with the UK general practice research database. BMJ 2007;335:982.

7 Gulliford MC, Moore MV, Little P, et al. Safety of reduced antibiotic prescribing for self limiting respiratory tract infections in primary care: cohort study using electronic health records. BMJ 2016;354:i3410-10.

8 Gharbi M, Drysdale JH, Lishman $\mathrm{H}$, et al. Antibiotic management of urinary tract infection in elderly patients in primary care and its association with bloodstream infections and all cause mortality: population based cohort study. BMJ 2019;364:I525.

9 Costelloe C, Metcalfe C, Lovering A, et al. Effect of antibiotic prescribing in primary care on antimicrobial resistance in individual patients: systematic review and meta-analysis. BMJ 2010;340:c2096.

10 Herrett E, Gallagher AM, Bhaskaran K, et al. Data resource profile: clinical practice research Datalink (CPRD). Int J Epidemiol 2015;44:827-36.

11 Jones KH, Ford DV, Lyons RA. The SAIL Databank: 10 years of spearheading data privacy and research utility, 2007-2017 Swansea University Medical School, Ysgol Feddygaeth Prifysgol Abertawe; 2017: 15.

12 University of Manchester, Institute of Population Health. ClinicalCodes Repository. Available: https://clinicalcodes.rss.mhs. man.ac.uk/ [Accessed 6 Feb 2020].

13 van Staa TP, Palin V, Li Y, et al. The effectiveness of frequent antibiotic use in reducing the risk of infection-related hospital admissions: results from two large population-based cohorts. BMC Med 2020;18:40.

14 Charlson ME, Pompei P, Ales KL, et al. A new method of classifying prognostic comorbidity in longitudinal studies: development and validation. J Chronic Dis 1987;40:373-83.
15 Government M of H Communities \& Local. English indices of multiple deprivation 2015: technical report, 2015. Available: https://www.gov. uk/government/publications/english-indices-of-deprivation-2015technical-report [Accessed 6 Feb 2020].

16 Babyak MA. Rescaling continuous predictors in regression models. statistical tips from the editors of psychosomatic medicine, 2009. Available: http://stattips.blogspot.com/2009/08/rescalingcontinuous-predictors-in.html [Accessed 6 Feb 2020].

17 Gordon M, Lumley T. Advanced Forest Plot Using 'grid' Graphics, 2017. Available: https://cran.r-project.org/web/packages/forestplot/ forestplot.pdf

18 Wickham H, François R, Henry L. A Grammar of Data Manipulation; Package 'dplyr', 2019. Available: https://cran.r-project.org/web/ packages/dplyr/dplyr.pdf

19 et alRipley B, Venables B, Bates DM. Support Functions and Datasets for Venables and Ripley's MASS, 2019. Available: https:// cran.r-project.org/web/packages/MASS/MASS.pdf

20 Hope EC, Crump RE, Hollingsworth TD, et al. Identifying English practices that are high antibiotic prescribers accounting for comorbidities and other legitimate medical reasons for variation. EClinicalMedicine 2018;6:36-41.

21 Ashworth M, Armstrong D. The relationship between general practice characteristics and quality of care: a national survey of quality indicators used in the UK quality and outcomes framework, 2004-5. BMC Fam Pract 2006;7:68.

22 Ashworth M, Seed P, Armstrong D, et al. The relationship between social deprivation and the quality of primary care: a national survey using indicators from the UK quality and outcomes framework. Br J Gen Pract 2007;57:441-8.

23 Yoshikawa TT. Epidemiology and unique aspects of aging and infectious diseases. Clin Infect Dis 2000;30:931-3.

24 Wehling M. Drug therapy for the elderly. Springer Science \& Business Media, 2012.

25 Lishman $\mathrm{H}$, Costelloe C, Hopkins S, et al. Exploring the relationship between primary care antibiotic prescribing for urinary tract infections, Escherichia coli bacteraemia incidence and antimicrobial resistance: an ecological study. Int J Antimicrob Agents 2018;52:790-8.

26 Little P, Gould C, Williamson I, et al. Reattendance and complications in a randomised trial of prescribing strategies for sore throat: the medicalising effect of prescribing antibiotics. BMJ 1997;315:350-2.

27 Butler CC, Simpson SA, Dunstan F, et al. Effectiveness of multifaceted educational programme to reduce antibiotic dispensing in primary care: practice based randomised controlled trial. BMJ 2012;344:d8173.

28 Royal College of General Practitioners. Target antibiotics: treat antibiotics Responsibly, guidance, education, tools, 2013. Available: http://www.rcgp.org.uk/clinical-and-research/toolkits/targetantibiotic-toolkit.aspx [Accessed 14 May 2018].

29 NHS England. Quality Premium : Guidance for 2017-19, 2018 Available: https://www.england.nhs.uk/ccg-out-tool/qual-prem/ [Accessed 14 May 2019].

30 Bou-Antoun S, Costelloe C, Honeyford K, et al. Age-Related decline in antibiotic prescribing for uncomplicated respiratory tract infections in primary care in England following the introduction of a national financial incentive (the quality premium) for health commissioners to reduce use of antibiotics in the community: an interrupted time series analysis. J Antimicrob Chemother 2018;73:2883-92.

31 Moore M, Stuart B, Lown M, et al. Predictors of adverse outcomes in uncomplicated lower respiratory tract infections. Ann Fam Med 2019;17:231-8

32 Little P, Stuart B, Hobbs FDR, et al. Antibiotic prescription strategies for acute sore throat: a prospective observational cohort study. Lancet Infect Dis 2014;14:213-9.

33 Spinks A, Glasziou PP, Del Mar CB. Antibiotics for sore throat. Cochrane Database Syst Rev 2013:CD000023.

34 Venekamp RP, Sanders SL, Glasziou PP, et al. Antibiotics for acute otitis media in children. Cochrane Database Syst Rev 2015;303.

35 Little P, Stuart B, Hobbs FDR, et al. Predictors of suppurative complications for acute sore throat in primary care: prospective clinical cohort study. BMJ 2013;347:f6867. 